\title{
Role of anti-Müllerian hormone and testosterone in follicular growth: a cross- sectional study
}

Ping-Ping Lv ${ }^{1 \dagger}$, Min Jin ${ }^{2+}$, Jin-Peng Rao ${ }^{2}$, Jian Chen², Li-Quan Wang ${ }^{2}$, Chang-Chang Huang ${ }^{2}$, Song-Qing Yang ${ }^{2}$, Qiu-Ping $\mathrm{YaO}^{2}$, Lei Feng ${ }^{3}$, Jin-Ming Shen ${ }^{3}$ and Chun Feng ${ }^{2^{*}}$ (i)

\begin{abstract}
Background: Anti-Müllerian hormone (AMH) is now considered the best serum biomarker of ovarian reserve, while basal sex hormones are classic markers used for assessing ovarian reserve. The interaction between AMH and sex hormones are complicated and not sufficiently addressed. In this study, we took diminished ovarian reserve (DOR) and polycystic ovarian syndrome (PCOS) as two extremes of ovarian reserve (deficient and excessive respectively) to investigate the role of $\mathrm{AMH}$ and sex hormones in follicular growth.

Methods: A retrospective cross-sectional survey was performed. The patients assessed AMH and basal sex hormones in the Second Hospital of Zhejiang University from April 2016 to March 2019 were involved in this study. Serum $\mathrm{AMH}$ and sex hormone concentrations were tested with electrochemiluminescence method. Stepwise linear regression and binary logistic regression was used to determine the predictors of $\mathrm{AMH}$ level and to explore the involved factors determining DOR and PCOS.

Results: In the present study, we found that age and follicle-stimulating hormone (FSH) were main negative correlation factors, and luteinizing hormone $(\mathrm{LH})$ and testosterone $(T)$ were main positive factors of $\mathrm{AMH}$. In DOR group, age, FSH and estradiol $\left(E_{2}\right)$ increased and T decreased, while in PCOS group, LH and T increased. Binary logistic regression found that age, weight, $\mathrm{FSH}, \mathrm{E}_{2}$, and T were the significant factors which independently predicted the likelihood of DOR, and that age, body mass index (BMI), AMH, LH, and T predicted the likelihood of PCOS.

Conclusions: Our study demonstrated that age, FSH, and T were factors that most closely correlated with AMH level, and T was involved in both DOR and PCOS. Since DOR and PCOS are manifested with insufficient AMH and excessive AMH respectively, it is suggested that total testosterone correlated with AMH closely and plays an important role in follicular growth. More attention should be given to testosterone level during controlled ovarian hyperstimulation $(\mathrm{COH})$ process.
\end{abstract}

Keywords: Anti-Müllerian hormone (AMH), Diminished ovarian reserve (DOR), Polycystic ovarian syndrome (PCOS), Follicle-stimulating hormone (FSH), Luteinizing hormone (LH), Testosterone

\footnotetext{
* Correspondence: doctorf@@zju.edu.cn

${ }^{\dagger}$ Ping-Ping Lv and Min Jin contributed equally to this work.

${ }^{2}$ The Second Affiliated Hospital of Zhejiang University School of Medicine, 88 Jiefang Road, Hangzhou 310009, Zhejiang, China

Full list of author information is available at the end of the article
}

(c) The Author(s). 2020 Open Access This article is licensed under a Creative Commons Attribution 4.0 International License, which permits use, sharing, adaptation, distribution and reproduction in any medium or format, as long as you give appropriate credit to the original author(s) and the source, provide a link to the Creative Commons licence, and indicate if changes were made. The images or other third party material in this article are included in the article's Creative Commons licence, unless indicated otherwise in a credit line to the material. If material is not included in the article's Creative Commons licence and your intended use is not permitted by statutory regulation or exceeds the permitted use, you will need to obtain permission directly from the copyright holder. To view a copy of this licence, visit http://creativecommons.org/licenses/by/4.0/. The Creative Commons Public Domain Dedication waiver (http://creativecommons.org/publicdomain/zero/1.0/) applies to the data made available in this article, unless otherwise stated in a credit line to the data. 


\section{Background}

Gonadal hormone anti-Müllerian hormone (AMH), a member of transforming growth factor- $\beta$ (TGF- $\beta$ ) superfamily, is a glycoprotein synthesized by granulosa cells [1]. It is now considered the best serum biomarker of ovarian reserve, reflecting the number of primordial follicles and its response to exogenous gonadotropins [2]. It is generally agreed that intra-individual variation of serum AMH is less than true biological variability $[3,4]$, and $\mathrm{AMH}$ level is not disturbed by menstrual cycles and exogenous sex steroids [5], so the test of AMH can be performed on any day of menstrual cycle, which is much more convenient than basal follicle-stimulating hormone (FSH) test. Nowadays AMH is widely used in menopause prediction, predicting pregnancy chances in infertility couples, management in IVF, and management of women with cancer [1].

Some studies investigated the relationship between $\mathrm{AMH}$ and gonadotropins. It was found that after gonadotropin-releasing hormone agonist ( $\mathrm{GnRHa}$ ) treatment $\mathrm{AMH}$ levels decreased significantly together with increase of FSH and luteinizing hormone $(\mathrm{LH})$, and then all hormone levels reversed, in which there was a moderate negative correlation between AMH and FSH [6]. However, others reported that after $\mathrm{GnRHa}$ administration $\mathrm{AMH}$ level changed significantly, and changes of AMH levels did not correlate with changes of gonadotropins, estradiol, or progesterone [7]. Evenmore, it was found that AMH receptor type 2 (AMHR2) was expressed in pituitary gonadotrope cells and AMH directly stimulated the secretion of FSH and $\mathrm{LH}$ [8-10]. In all, the relationship between $\mathrm{AMH}$ and sex hormones is complicated and inconsistent, further investigation is required to address the correlation.

$\mathrm{AMH}$ inhibits follicle development in two critical points: the recruitment of primordial follicles into the pool of growing follicles and the responsiveness of growing follicles to FSH [11-13]. An assessment of the correlation of $\mathrm{AMH}$ and individual sex hormone would be valuable for understanding the regulatory function of $\mathrm{AMH}$ in follicle development. Diminished ovarian reserve (DOR) and polycystic ovarian syndrome (PCOS) are representative of two extremes of ovarian reserve $[14,15]$, to explore the predictive factors of DOR and PCOS helps to understand the regulation mechanism of follicle development.

The aim of the present study was to determine the association between serum AMH level and serum basal sex hormone concentrations. Firstly, correlation analysis was performed to explore the individual correlation between $\mathrm{AMH}$ and basal sex hormones, and further multiple regression analysis was carried out to select predictive determinants of serum AMH level. Secondly, sex hormone levels were compared in DOR and PCOS populations, and logistic regression analysis was used to further determine the predictive factors of DOR and PCOS.

\section{Methods}

\section{Study population}

This was a retrospective cross-sectional study for correlation between $\mathrm{AMH}$ and sex hormones in women attending for pre-pregnancy examination or infertility at the Second Hospital of Zhejiang University School of Medicine. This study adhered to standard biosecurity and institutional safety procedures. As shown in Fig. 1, the patients performed AMH and basal sex hormones examination from April 2016 to March 2019 were included in this study. The specialists of reproductive medicine or gynecology recorded the demographic characteristics, carried out the examinations, and made diagnosis. Exclusion criteria included: 1) ovarian cysts, 2) hyperprolactinemia, 3) malignant diseases, 4) surgeries, 5) hypophysoma, and 6) diabetes mellitus.

\section{Outcome measurements}

Demographic characteristics and diagnosis were recorded in the database system. Serum AMH and sex hormones levels were measured with electrochemiluminescence method by Elecsys ${ }^{\oplus}$ AMH, FSH, LH, prolactin (PRL), estradiol $\left(\mathrm{E}_{2}\right)$, progesterone $(\mathrm{P})$, testosterone $(\mathrm{T})$ from Roche Diagnostics. Fasting plasma glucose (FPG) was determined by commercial enzymatic methods from Shanghai Rongsheng Biotech. Fasting plasma insulin (FPI) was determined with chemiluminescence from SIEMENs Healthcare Diagnostics. AMH is an effective test predicting ovarian reserve, and low AMH cutpoint (0.2-0.7 ng/ $\mathrm{ml}$ ) have been found to have ideal sensitivity and specificity for DOR [16]. In the present study diagnosis of DOR was made when AMH was less than $0.7 \mathrm{ng} / \mathrm{ml}$. According to Chinese Guidelines for Diagnosis and Treatment of Polycystic Ovary Syndrome (Chinese Society of Obstetrics and Gynecology Endocrinology Group and Guidelines Expert Group, 2018) [17], the diagnosis of PCOS was based on the presence of oligomenorrhea/amenorrhea, and at least one of the following criteria: hyperandrogenaemia/ hyperandrogenism and polycystic ovaries.

\section{Statistics}

Analyses were performed with the use of SPSS 19.0 statistics package (SPSS, Chicago, IL, USA). Continuous variables were presented as mean values \pm SD. One-way ANOVA was used for compares between groups, and Tukey post hoc pairwise comparison was performed with significant difference. Pearson correlation analysis was adopted to analyze the relationship between two variables. Stepwise linear regression was used to determine the significant predictors of serum AMH level. Binary logistic regression with forward method was conducted to explore the involved factors determining DOR and PCOS. A $P$ value of $<0.05$ was considered as statistically significant. 


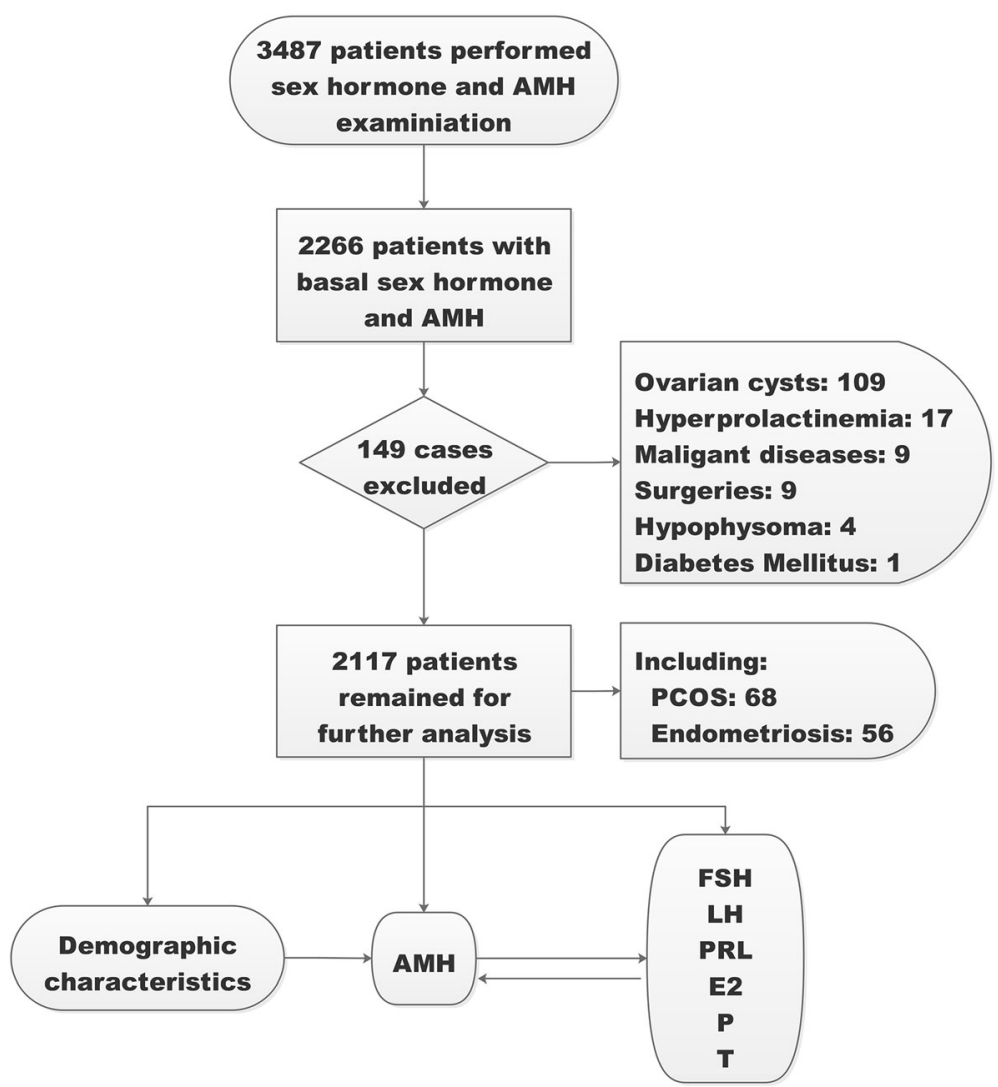

Fig. 1 Work flow. Of 3487 women with both AMH and sex hormones assessment, 1221 cases were excluded because the sex hormones assessment was not performed in early follicle phase, and 149 cases were excluded for ovarian cysts, hyperprolactinemia and so on. Finally 2117 women were remained for further analyses. PCOS: polycystic ovarian syndrome. AMH: anti-Müllerian hormone. FSH: follicle-stimulating hormone. LH: luteinizing hormone. PRL: prolactin. E2: estradiol. P: progesterone. T: testosterone

\section{Results}

\section{Description of the study sample}

As shown in Fig. 1, from April 2016 to March 2019 a total of 3487 women examined with AMH and sex hormones were included. One thousand two hundred twenty-one cases were excluded because the sex hormone test was not taken in early follicle phase. Later 149 cases were excluded for ovarian cysts, hyperprolactinemia, malignant diseases, surgeries, hypophysoma, and diabetes mellitus. Finally 2117 women were remained for further analysis of correlation, including 68 PCOS and 56 endometriosis.

The characteristics of the study population are presented in Table 1. In the DOR group there were

Table 1 Characteristics of the included subjects

\begin{tabular}{|c|c|c|c|c|c|}
\hline Characteristics & All & Control & DOR & PCOS & $P$ \\
\hline Cases (N) & 2117 & 1714 & 335 & 68 & \\
\hline Age (years) & $32.02 \pm 6.80$ & $30.51 \pm 5.54$ & $40.67 \pm 6.55$ & $27.71 \pm 3.43$ & $<0.001$ \\
\hline Weight (kg) & $54.34 \pm 8.01$ & $53.83 \pm 7.99$ & $56.06 \pm 7.39$ & $58.83 \pm 9.14$ & $<0.001$ \\
\hline Height (cm) & $160.90 \pm 4,81$ & $160.94 \pm 4.85$ & $160.62 \pm 4.65$ & $161.22 \pm 4.60$ & 0.455 \\
\hline $\mathrm{BMI}\left(\mathrm{kg} / \mathrm{m}^{2}\right)$ & $20.98 \pm 2,86$ & $20.77 \pm 2.84$ & $21.72 \pm 2.63$ & $22.60 \pm 3.19$ & $<0.001$ \\
\hline $\mathrm{AMH}(\mathrm{ng} / \mathrm{ml})$ & $3.48 \pm 3.09$ & $4.08 \pm 3.00$ & $0.29 \pm 0.22$ & $6.93 \pm 3.84$ & $<0.001$ \\
\hline FPG (mmol/L) & $5.01 \pm 0.29$ & $5.02 \pm 0.30$ & $4.99 \pm 0.28$ & $4.97 \pm 0.28$ & 0.288 \\
\hline $\mathrm{FPI}(\mathrm{pmol} / \mathrm{L})$ & $90.28 \pm 66.66$ & $91.31 \pm 67.48$ & $75.80 \pm 55.39$ & $96.14 \pm 54.34$ & 0.565 \\
\hline
\end{tabular}

Data were presented as mean \pm SD

DOR Decreased ovarian reserve. AMH Anti-Müllerian hormone. PCOS Polycystic ovarian syndrome. BMI Body mass index. FPG Fasting plasma glucose. FPI Fasting plasma insulin 
higher mean age, weight and BMI $(P<0.001)$. In PCOS group the mean age was significantly lower in PCOS group than in control group, and the mean weight, BMI, and $\mathrm{AMH}$ were significantly higher $(\mathrm{P}<$ 0.001). There was no difference in height, FPG, and FPI levels $(P>0.05)$.

\section{Correlation between demographic characteristics, sex hormones and AMH}

Pearson test was carried out to investigate the correlation between demographic characteristics, gonadotropins, ovarian steroids and $\mathrm{AMH}$. As shown in Fig. 2a and $\mathrm{d}$, in total population, age $(\mathrm{R}=-0.457$, $P<0.001)$ and FSH $(\mathrm{R}=-0.310, \quad P<0.001)$ were moderately and mildly correlated with $\mathrm{AMH}$ negatively. Meanwhile, LH $(\mathrm{R}=0.307, \mathrm{P}<0.001)$ and $\mathrm{T}$ $(\mathrm{R}=0.346, \quad \mathrm{P}<0.001)$ were mildly correlated with
AMH positively (Fig. 2e and i). There were weak negative correlation between weight $(R=-0.050, P=$ $0.021)$, BMI $(\mathrm{R}=-0.058, P=0.008), \mathrm{E}_{2} \quad(\mathrm{R}=-0.052$, $P=0.017$ ), and AMH (Fig. 2b, c, and g). There was no correlation between PRL, $\mathrm{P}$, height and $\mathrm{AMH}$ $(P>0.01)$.

Since Pearson correlation analyses indicated that age, weight, BMI, FSH, $\mathrm{LH}, \mathrm{E}_{2}$, and $\mathrm{T}$ were correlated with $\mathrm{AMH}$, multiple linear regression analyses with stepwise analyses were performed to further address the relationship between these indicators and $\mathrm{AMH}$. The results showed that in the control group, significant contributions for the prediction of serum AMH were provided by age, FSH, $\mathrm{LH}, \mathrm{E}_{2}$, and $\mathrm{T}$ but not weight and BMI (Table 2). Age, FSH, and $\mathrm{E}_{2}$ negatively correlated with $\mathrm{AMH}$ and while $\mathrm{LH}$ and $\mathrm{T}$ positively correlated with AMH $(P<0.001)$.

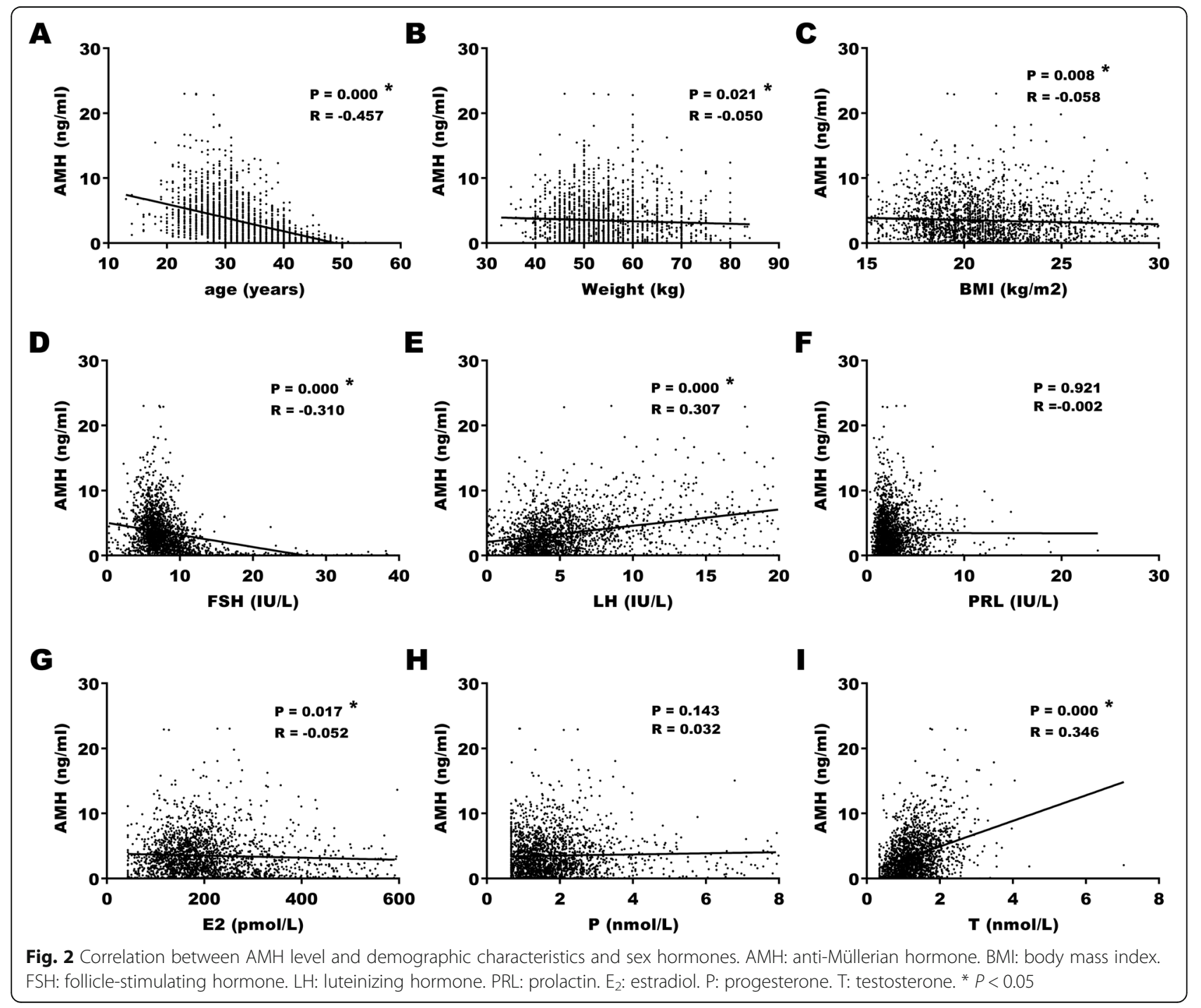


Table 2 Correlation of demographic characteristics, sex hormones, and $\mathrm{AMH}$ by multiple linear regression

\begin{tabular}{llllll}
\hline Variables & B & Standard error & LCL & UCL & $P$ value \\
\hline age & -0.117 & 0.009 & -0.134 & -0.100 & $<0.001$ \\
LH & 0.292 & 0.016 & 0.260 & 0.323 & $<0.001$ \\
FSH & -0.199 & 0.012 & -0.223 & -0.175 & $<0.001$ \\
$E_{2}$ & -0.005 & 0.001 & -0.006 & -0.004 & $<0.001$ \\
T & 0.857 & 0.106 & 0.649 & 1.065 & $<0.001$ \\
\hline
\end{tabular}

LCL 95\% lower confidence limit. UCL 95\% upper confidence limit. $L H$ Luteinizing hormone. FSH Follicle-stimulating hormone. $E_{2}$ Estradiol. $T$ Testosterone

\section{Demographic characteristics and sex hormones in DOR group}

Age was significantly older in DOR group in comparison to control (Fig. 3a). Since DOR was diagnosed with $\mathrm{AMH}$ cutoff in this study, the AMH level in DOR group was much lower than control group (Fig. 3b). Weight and BMI showed slightly increase in DOR group $(P<$ 0.05) (Fig. 3c and d). For pituitary hormones, FSH level increased significantly in DOR group, while LH and PRL did not alter significantly between two groups $(P>0.05)$ (Fig. 3e, f, and g). For ovarian steroids, a significant increase of $E_{2}$ and a significant decrease of $T$ was observed in DOR groups when compared to control (Fig. 3h, h, and $\mathrm{j}$ ).

\section{Demographic characteristics and sex hormones in PCOS group}

Age was significantly lower in PCOS group compared with control (Fig. 3a). As shown in Fig. 3b, mean AMH level nearly doubled in PCOS group compared to control group $(6.93 \pm 3.84$ vs. $4.08 \pm 3.00, P<0.05)$. Weight and BMI levels revealed a significant increase in PCOS group when compared with control (Fig. 3c and d). For pituitary hormones, LH level increased significantly in PCOS group, and FSH and PRL levels were similar between the two groups (Fig. 3e, f and g). For ovarian hormones, $\mathrm{T}$ level showed a significant rise in PCOS group when compared to control, and variation in $E_{2}$ and $P$ were not significant (Fig. 3h, i, and j).

\section{Logistic regression of DOR and PCOS groups}

Binary logistic regression with forward method was used to analyze the prediction on DOR by age, weight, BMI, $\mathrm{FSH}, \mathrm{E}_{2}$ and $\mathrm{T}$. Age, weight, FSH, $\mathrm{E}_{2}$, and $\mathrm{T}$, but not $\mathrm{BMI}$, were the significant factors which independently predicted the likelihood of DOR. In the logistic regression model adjusted for age, weight, $\mathrm{FSH}$ and $\mathrm{E}_{2}$, one $\mathrm{nmol} / \mathrm{L}$ increase of $\mathrm{T}$ level was associated with 0.60 times decreased (95\% CI: $0.42-0.87, P=0.007$ ) risk of DOR (Table 3).

Binary logistic regression with forward method was used to analyze the prediction on PCOS by age, weight,
BMI, AMH, FSH, LH and T. Age, BMI, AMH, LH, and $\mathrm{T}$, but not weight and FSH, were the significant factors which independently predicted the likelihood of PCOS. In a logistic regression model adjusted for age, BMI, $\mathrm{AMH}$ and $\mathrm{LH}$, one $\mathrm{nmol} / \mathrm{L}$ increase of $\mathrm{T}$ level was associated with 1.54 times increased (95\% CI: 1.06-2.24, $P=$ 0.025 ) risk of PCOS (Table 3 ).

\section{Discussion}

In this study we demonstrated that age was moderately negatively correlated with $\mathrm{AMH}$ concentration. Risk of DOR increased and risk of PCOS decreased significantly with age. Since it is widely accepted that oocyte number and quality decline with age $[16,18]$, it is reasonable that age is a dominant factor of DOR. However, PCOS is considered a lifelong disease. What is the underlying reason for the decreased risks in aged population? In PCOS patients, due to the increased number of antral follicles, there is higher $\mathrm{AMH}$ concentration and lower basal FSH concentration compared to women with normal cycle [19], which leads to rare or no ovulation in PCOS. Aging leads to decreased follicle number, with decreased AMH concentration and increased basal FSH level, which is in favor of follicular growth and regular ovulation [20]. Therefore, it is possible that the diagnosis of PCOS cannot be made any more if it is not diagnosed in earlier age, so the incidence of PCOS decreased as age increased. In younger women with PCOS the main manifestations are hyperandrogenism and chronic anovulation, whereas in older women with PCOS, the main manifestations are obesity, insulin resistance, and metabolic disturbances [21]. A study comparing postmenopausal women with and without PCOS found that PCOS women had similar menopausal age, body weight, BMI, LH, total testosterone, and estradiol, except lower FSH [22]. In a word, the decreased risk of PCOS in aged population may be due to the difficulty of diagnosis.

In the present study, basal FSH was mildly correlated with AMH negatively. This result is in line with the previous studies. It is reported that there is a negative correlation between $\mathrm{AMH}$ concentration and serum $\mathrm{FSH}$ concentration in PCOS, and AMH may reduce the concentration of FSH and decrease follicle sensitivity to FSH by inhibition of aromatase [23, 24]. This study demonstrates that elevated FSH level is associated with increased risk of DOR, and this is generally accepted worldwide [16].

In this study basal LH was mildly correlated with AMH positively, and elevated LH level was associated with increased risk of PCOS. This result is consistent with the previous studies [25-27], indicating an 

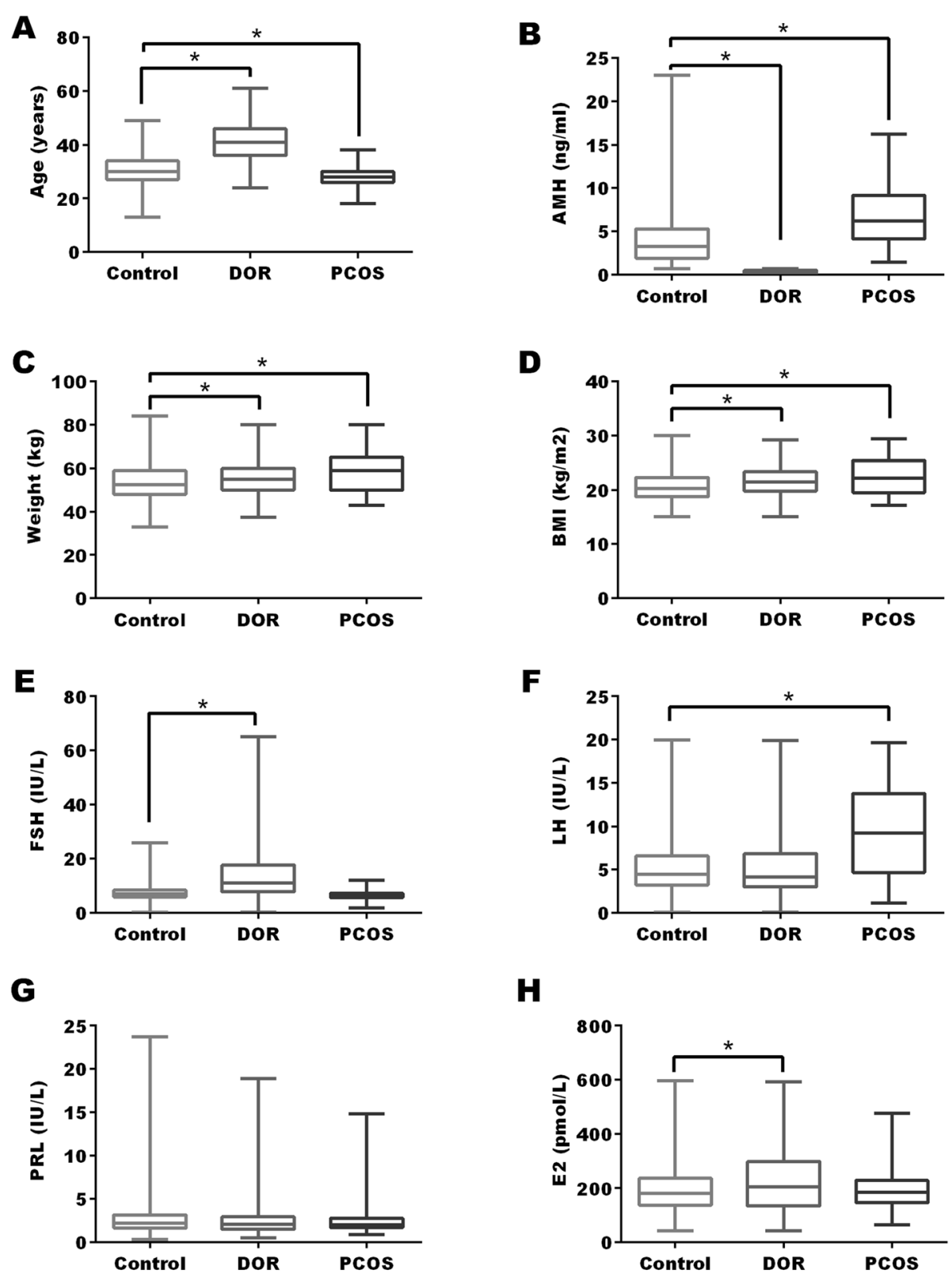

H
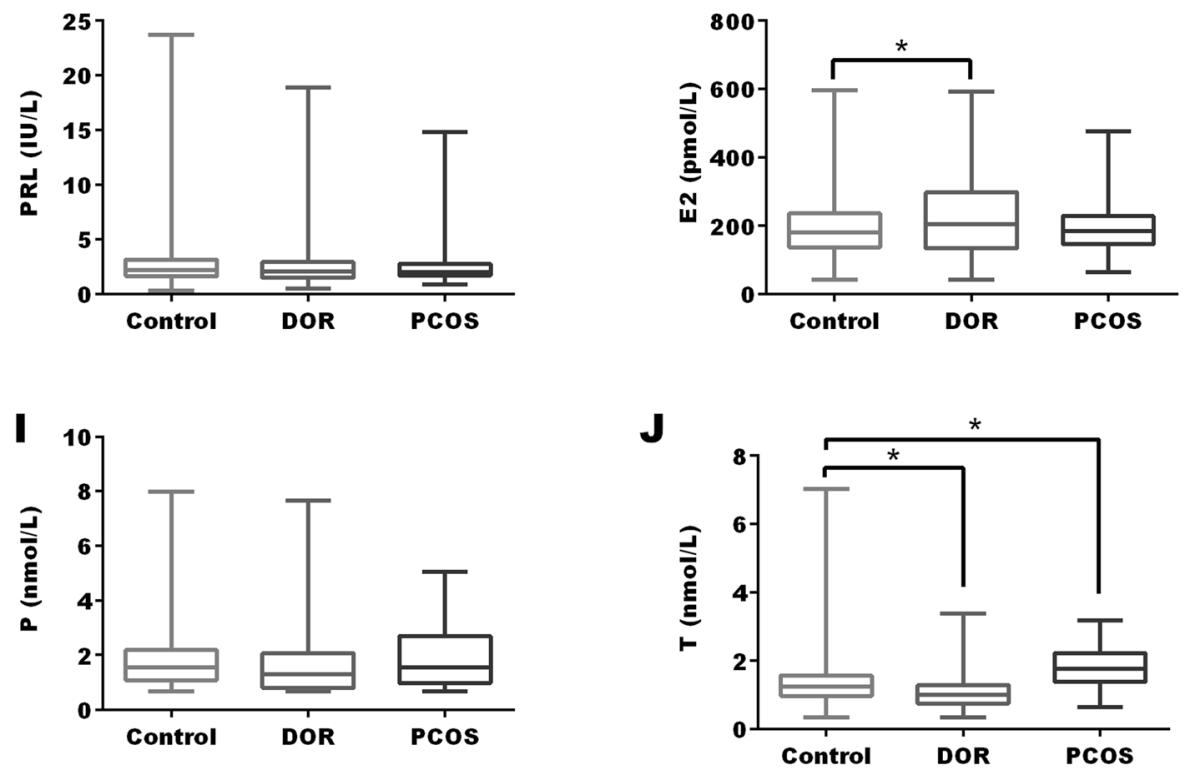

Fig. 3 Demographic characteristics and sex hormones in NOR, DOR, and PCOS groups. NOR: normal ovarian reserve. DOR: diminished ovarian reserve. PCOS: polycystic ovarian syndrome. BMl: body mass index. FSH: follicle-stimulating hormone. LH: luteinizing hormone. PRL: prolactin. $E_{2}$ : estradiol. P: progesterone. T: testosterone. ${ }^{*} P<0.05$ 
Table 3 Relationship of $\mathrm{AMH}$, demographic characteristics, and sex hormones in DOR and PCOS patients using logistic regression

\begin{tabular}{|c|c|c|c|c|c|c|c|}
\hline \multicolumn{4}{|l|}{ DOR } & \multicolumn{4}{|l|}{ PCOS } \\
\hline Variables & OR & $95 \% \mathrm{Cl}$ & $P$ value & Variables & OR & $95 \% \mathrm{Cl}$ & $P$ value \\
\hline age & 1.25 & $1.22-1.29$ & $<0.001$ & age & 0.92 & $0.88-0.97$ & 0.002 \\
\hline weight & 1.02 & $1.00-1.05$ & 0.041 & $\mathrm{BMI}$ & 1.23 & $1.14-1.34$ & $<0.001$ \\
\hline FSH & 1.32 & $1.25-1.38$ & $<0.001$ & $\mathrm{AMH}$ & 1.12 & $1.05-1.19$ & 0.001 \\
\hline$E_{2}$ & 1.00 & $1.00-1.01$ & $<0.001$ & $\mathrm{LH}$ & 1.14 & $1.08-1.20$ & $<0.001$ \\
\hline $\mathrm{T}$ & 0.60 & $0.42-0.87$ & 0.007 & $\mathrm{~T}$ & 1.54 & $1.06-2.24$ & 0.025 \\
\hline
\end{tabular}

DOR Diminished ovarian reserve. AMH Anti-Müllerian hormone. PCOS Polycystic ovarian syndrome. BMI Body mass index. $\mathrm{LH}$ Luteinizing hormone. FSH Follicle-stimulating hormone. $E_{2}$ Estradiol. $T$ Testosterone

interaction between $\mathrm{AMH}$ and $\mathrm{LH}$ secretion, which may contribute to the pathogenesis of PCOS [28]. On one hand, AMH activates the GnRH neuron through AMH receptors and increases GnRH-dependent LH pulsatility and secretion [10]. On the other hand, LH may increase AMH production in granulosa cells [29]. Therefore, increased $\mathrm{AMH}$ and $\mathrm{LH}$ stimulate each other mutually and play a role together in follicular arrest and pathogenesis of PCOS.

We found that $\mathrm{T}$ was mildly correlated with $\mathrm{AMH}$ positively. Moreover, elevated $\mathrm{T}$ level was associated with increased risk of PCOS and decreased risk of DOR. The positive correlation between serum AMH and total testosterone has been reported previously $[24-26,30]$. It is demonstrated that androgens stimulate follicular FSHR expression, amplify FSH effect, promote follicular growth, which may lead to increased AMH production [31, 32]. Meanwhile, elevated $\mathrm{AMH}$ increases $\mathrm{LH}$ secretion in GnRH-neurone through AMH receptors [10], and elevated $\mathrm{AMH}$ inhibites aromatase expression in granulosa cells disturbing the transformation of androgen to estrogen $[13,33]$, which leads to the elevated androgen level. The two hormones may mutually reinforce each other, which may be the reason for the positive correlation of them. Elevated $\mathrm{T}$ level is associated with increased risk of PCOS. In PCOS with high androgen, serum AMH levels increase significantly compared with PCOS with normal androgen [27]. Others suggest that serum AMH level is related to the severity of PCOS $[34,35]$. Such an interaction may contribute to the pathogenesis of PCOS [28].

As shown in Fig. 4, in PCOS high androgen leads to increased AMH level, and high AMH inhibits follicular development, leading to accumulation of small follicles without development of dominant follicles. High AMH decreases expression of FSHR and inhibit the response to exogenous gonadotropin, which leads to the poor response of ovarian stimulation. On one hand AMH impedes the conversion of androgen to estrogen, and on the other hand accumulated androgen leads to the increase of $\mathrm{AMH}$, which thus initiates a vicious cycle. In DOR women, reduced androgen concentration accompanies with decreased AMH level. Insufficient androgen level cannot supply sufficient substrates for ovarian steroids synthesis. Meanwhile, decreased AMH level leads to accelerated follicular consumption and reduced ovarian reserve.

Either excess or deficiency of androgen may do harm to follicle development and deteriorate fertility [36]. In PCOS, it is demonstrated that suppression of androgen can improve the results of ovulation induction in PCOS $[37,38]$. In DOR, androgen supplement may lead to a PCOS-like phenotype that increases oocyte production. Some randomized controlled trials (RCTs) have shown that DHEA and testosterone supplement can improve fertility in women with DOR $[39,40]$. It is estimated that about a quarter of women with DOR are treated with DHEA supplements in all IVF centers [41]. Both literatures and our study point out that appropriate androgen level is required for normal follicular growth and is important in controlled ovarian hyperstimulation $(\mathrm{COH})$ process.

The present study has some limitations. Firstly, in this study the diagnosis of DOR was made based on solely AMH. Until now, there is no uniformly accepted definition of DOR, as stated in the committee opinion provided by American Society for Reproductive Medicine [16]. There is mounting evidence to support the use of AMH as a screening test for poor ovarian response, but the data are insufficient yet [16]. In the Bologna criteria abnormal ovarian reserve test included AFC less than 5-7 follicles or AMH below $0.5-1.1 \mathrm{ng} / \mathrm{ml}$ [42]. The definition of DOR might be more solid with more criteria such as AFC. Secondly, since this is a retrospective observational study, we cannot distinguish other factors participating in follicular growth. Further RCTs and fundamental studies are required to confirm the roles of $\mathrm{T}$ in follicular growth and explore the underlying mechanisms. Furthermore, in infertile population with IVF we can develop an algorithm using $\mathrm{AMH}$ and $\mathrm{T}$ to predict ovarian response, which can help in daily work.

\section{Conclusions}

In the present study, we found that age and FSH were main negative correlation factors, and $\mathrm{LH}$ and $\mathrm{T}$ were main positive factors of AMH. In DOR group, age, FSH and $E_{2}$ increased and $T$ decreased, while in PCOS group, $\mathrm{LH}$ and $\mathrm{T}$ increased and FSH decreased. Binary logistic regression found that age, weight, FSH, $E_{2}$, and $T$ were the significant factors which 


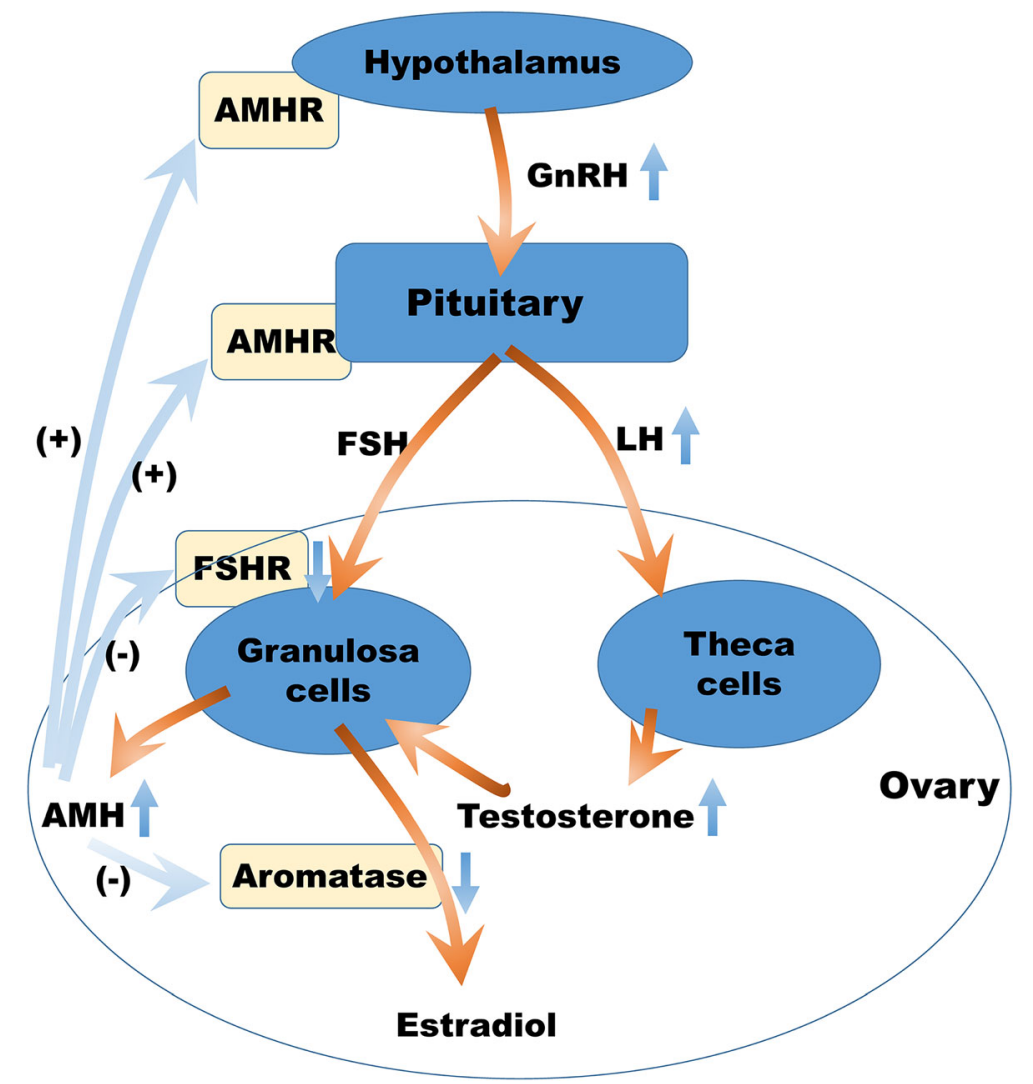

Fig. 4 Schematic diagram of AMH and androgen in DOR and PCOS. In PCOS, elevated AMH stimulates LH expression by AMHR on hypothalamus and pituitary, increasing androgen secretion of theca cells. Meanwhile, elevated AMH inhibits FSHR expression and aromatase expression, which prevents the conversion of androgen to estrogen and follicle growth, leading to increase of androgen level. In return, elevated androgen stimulates AMH secretion of granulosa cells directly and indirectly. Therefore, elevated AMH and androgen promote mutually in a circle. In DOR, decreased AMH removes AMH induced inhibition of FSH expression, leading to faster conversion of androgen to estrogen and accelerated follicle consumption, which results in insufficient androgen and decreased ovarian reserve. In return, decreased androgen leads to decreased estradiol, elevated FSH, accelerated follicle consumption, and decreased AMH. AMH: anti-Müllerian hormone. DOR: diminished ovarian reserve. PCOS: polycystic ovarian syndrome. AMHR: receptor of AMH. FSH: follicle-stimulating hormone. LH: luteinizing hormone

independently predicted the likelihood of DOR, and that age, BMI, AMH, LH, and T predicted the likelihood of PCOS. Since DOR and PCOS are manifested with insufficient and excessive $\mathrm{AMH}$ respectively, our study demonstrated that age and $\mathrm{T}$ were factors that most closely correlated with AMH level, and involved in DOR and PCOS. It is suggested that total testosterone plays an important role in follicular growth and more attention should be given to total testosterone level during $\mathrm{COH}$ process.

\section{Abbreviations}

AMH: Anti-Müllerian hormone; AMHR2: AMH receptor type 2; BMI: Body mass index; $\mathrm{COH}$ : Controlled ovarian hyperstimulation; DOR: Diminished ovarian reserve; $E_{2}$ : Estradiol; FPG: Fasting plasma glucose; FPI: Fasting plasma insulin; FSH: Follicle-stimulating hormone; GnRHa: Gonadotropin-releasing hormone agonist; LH: Luteinizing hormone; P: Progesterone; PCOS: Polycystic ovarian syndrome; PRL: Prolactin; T: Testosterone; TGF- $\beta$ : Transforming growth factor$\beta$

\section{Acknowledgements}

We are especially grateful to Ya-Wen Xing for his kind help in data collection.

\section{Authors' contributions}

CF and JMS conceived and designed the study; PPL, MJ, JPR, LQW, JC, CCH, SQY, QPY, LF and JMS collected and analyzed the data; PPL and JMS organized and wrote the manuscript. All authors read and approved the final manuscript.

\section{Funding}

This work was financially supported by National Key R\&D Program of China (2018YFC1004900 to CF) and National Natural Science Foundation of China (81871176 to CF, 81701461 to PPL, and 81671487 to MJ). The funders covered publication fee. None of the funders were involved in the design of the study, in collection, analysis, and interpretation of data, and in writing the manuscript.

\section{Availability of data and materials}

The datasets used and/or analyzed during the current study are available from the corresponding author on reasonable request. 


\section{Ethics approval and consent to participate}

The present study was approved by the Ethics Committee of the Second Affiliated Hospital of Zhejiang University School of Medicine (IR2019001059). Because this was a retrospectively observational study with no intervention, formal ethical approval and written consent were not required.

\section{Consent for publication}

Not applicable.

\section{Competing interests}

The authors declare that they have no competing interests.

\section{Author details}

'The Women's Hospital of Zhejiang University School of Medicine, Hangzhou 310006, Zhejiang, China. ${ }^{2}$ The Second Affiliated Hospital of Zhejiang University School of Medicine, 88 Jiefang Road, Hangzhou 310009, Zhejiang, China. ${ }^{3}$ The First Affiliated Hospital of Zhejiang Chinese Medicine University, Hangzhou 310006, Zhejiang, China.

\section{Received: 18 March 2020 Accepted: 8 June 2020}

\section{Published online: 08 July 2020}

\section{References}

1. Broer SL, Broekmans FJ, Laven JS, Fauser BC. Anti-Müllerian hormone: ovarian reserve testing and its potential clinical implications. Hum Reprod Update. 2014;20(5):688-701.

2. Hansen KR, Hodnett GM, Knowlton N, Craig LB. Correlation of ovarian reserve tests with histologically determined primordial follicle number. Fertil Steril. 2011:95(1):170-5.

3. van Disseldorp J, Lambalk CB, Kwee J, et al. Comparison of inter- and intracycle variability of anti-Mullerian hormone and antral follicle counts. Hum Reprod. 2010;25(1):221-7.

4. Fanchin R, Taieb J, Lozano DH, Ducot B, Frydman R, Bouyer J. High reproducibility of serum anti-Mullerian hormone measurements suggests a multi-staged follicular secretion and strengthens its role in the assessment of ovarian follicular status. Hum Reprod. 2005;20(4):923-7.

5. Deb S, Campbell BK, Pincott-Allen C, Clewes JS, Cumberpatch G, RaineFenning NJ. Quantifying effect of combined oral contraceptive pill on functional ovarian reserve as measured by serum anti-Müllerian hormone and small antral follicle count using three-dimensional ultrasound. Ultrasound Obstet Gynecol. 2012;39(5):574-80.

6. van Helden J, Evliyaoglu O, Weiskirchen R. Has GnRH a direct role in $\mathrm{AMH}$ regulation. Clin Endocrinol. 2019;90(6):827-33.

7. Su HI, Maas K, Sluss PM, Chang RJ, Hall JE, Joffe H. The impact of depot $\mathrm{GnRH}$ agonist on AMH levels in healthy reproductive-aged women. J Clin Endocrinol Metab. 2013;98(12):E1961-6.

8. Kereilwe $\mathrm{O}$, Pandey K, Borromeo V, Kadokawa H. Anti-Müllerian hormone receptor type 2 is expressed in gonadotrophs of postpubertal heifers to control gonadotrophin secretion. Reprod Fertil Dev. 2018;30(9):1192-203.

9. Bédécarrats GY, O'Neill FH, Norwitz ER, Kaiser UB, Teixeira J. Regulation of gonadotropin gene expression by Mullerian inhibiting substance. Proc Natl Acad Sci U S A. 2003;100(16):9348-53.

10. Cimino I, Casoni F, Liu X, et al. Novel role for anti-Müllerian hormone in the regulation of $\mathrm{GnRH}$ neuron excitability and hormone secretion. Nat Commun. 2016;7:10055.

11. Durlinger AL, Visser JA, Themmen AP. Regulation of ovarian function: the role of anti-Müllerian hormone. Reproduction. 2002;124(5):601-9.

12. Dewailly D, Robin G, Peigne M, Decanter C, Pigny P, Catteau-Jonard S. Interactions between androgens, FSH, anti-Müllerian hormone and estradiol during folliculogenesis in the human normal and polycystic ovary. Hum Reprod Update. 2016;22(6):709-24.

13. Pellatt $L$, Rice $S$, Dilaver N, et al. Anti-Müllerian hormone reduces follicle sensitivity to follicle-stimulating hormone in human granulosa cells. Fertil Steril. 2011;96(5):1246-51.e1.

14. Gleicher N, Weghofer A, Barad DH. Anti-Müllerian hormone (AMH) defines, independent of age, low versus good live-birth chances in women with severely diminished ovarian reserve. Fertil Steril. 2010;94(7):2824-7.

15. Tremellen K, Zander-Fox D. Serum anti-Mullerian hormone assessment of ovarian reserve and polycystic ovary syndrome status over the reproductive lifespan. Aust N Z J Obstet Gynaecol. 2015;55(4):384-9.
16. Practice Committee of the American Society for Reproductive Medicine. Testing and interpreting measures of ovarian reserve: a committee opinion. Fertil Steril. 2015;103(3):e9-e17..

17. group Csooagegage. Chinese guidelines for diagnosis and treatment of polycystic ovary syndrome. Chinese J Obstetr Gynecol. 2018;53(1):2-6.

18. Female age-related fertility decline. Committee Opinion. Fertil Steril. 2014; 101(3):633-4.

19. Hudecova M, Holte J, Olovsson M, Sundström PI. Long-term follow-up of patients with polycystic ovary syndrome: reproductive outcome and ovarian reserve. Hum Reprod. 2009;24(5):1176-83.

20. Beltadze K, Barbakadze L. Ovarian reserve in women of late reproductive age by the method of treatment of PCOS. Iran J Reprod Med. 2015;13(5):263-8.

21. Hsu MI. Changes in the PCOS phenotype with age. Steroids. 2013;78(8):761-6.

22. Schmidt J, Brännström M, Landin-Wilhelmsen K, Dahlgren E. Reproductive hormone levels and anthropometry in postmenopausal women with polycystic ovary syndrome (PCOS): a 21-year follow-up study of women diagnosed with PCOS around 50 years ago and their age-matched controls. J Clin Endocrinol Metab. 2011;96(7):2178-85.

23. Pellatt $L$, Rice $S$, Mason HD. Anti-Müllerian hormone and polycystic ovary syndrome: a mountain too high. Reproduction. 2010;139(5):825-33.

24. Pigny P, Merlen E, Robert $Y$, et al. Elevated serum level of anti-mullerian hormone in patients with polycystic ovary syndrome: relationship to the ovarian follicle excess and to the follicular arrest. J Clin Endocrinol Metab. 2003;88(12):5957-62

25. Pierre A, Peigné $M$, Grynberg M, et al. Loss of LH-induced down-regulation of anti-Müllerian hormone receptor expression may contribute to anovulation in women with polycystic ovary syndrome. Hum Reprod. 2013; 28(3):762-9.

26. Homburg R, Ray A, Bhide $P$, et al. The relationship of serum anti-Mullerian hormone with polycystic ovarian morphology and polycystic ovary syndrome: a prospective cohort study. Hum Reprod. 2013;28(4):1077-83.

27. Sova $H$, Unkila-Kallio L, Tiitinen $A$, et al. Hormone profiling, including antiMüllerian hormone (AMH), for the diagnosis of polycystic ovary syndrome (PCOS) and characterization of PCOS phenotypes. Gynecol Endocrinol. 2019; 35:1-6.

28. Garg D, Tal R. The role of AMH in the pathophysiology of polycystic ovarian syndrome. Reprod BioMed Online. 2016;33(1):15-28.

29. Pellatt $L$, Hanna L, Brincat $M$, et al. Granulosa cell production of antiMüllerian hormone is increased in polycystic ovaries. J Clin Endocrinol Metab. 2007;92(1):240-5

30. Tal R, Seifer DB, Khanimov M, Malter HE, Grazi RV, Leader B. Characterization of women with elevated antimüllerian hormone levels (AMH): correlation of $\mathrm{AMH}$ with polycystic ovarian syndrome phenotypes and assisted reproductive technology outcomes. Am J Obstet Gynecol. 2014;211(1):59. e1-8.

31. Vendola KA, Zhou J, Adesanya OO, Weil SJ, Bondy CA. Androgens stimulate early stages of follicular growth in the primate ovary. J Clin Invest. 1998; 101(12):2622-9.

32. Weil S, Vendola K, Zhou J, Bondy CA. Androgen and follicle-stimulating hormone interactions in primate ovarian follicle development. J Clin Endocrinol Metab. 1999;84(8):2951-6.

33. Grossman MP, Nakajima ST, Fallat ME, Siow Y. Müllerian-inhibiting substance inhibits cytochrome P450 aromatase activity in human granulosa lutein cell culture. Fertil Steril. 2008;89(5 Suppl):1364-70.

34. Jacob SL, Field HP, Calder N, Picton HM, Balen AH, Barth JH. Anti-Müllerian hormone reflects the severity of polycystic ovary syndrome. Clin Endocrinol. 2017:86(3):395-400.

35. Fraissinet A, Robin G, Pigny P, Lefebvre T, Catteau-Jonard S, Dewailly D. Use of the serum anti-Müllerian hormone assay as a surrogate for polycystic ovarian morphology: impact on diagnosis and phenotypic classification of polycystic ovary syndrome. Hum Reprod. 2017;32(8):1716-22.

36. Lebbe M, Woodruff TK. Involvement of androgens in ovarian health and disease. Mol Hum Reprod. 2013;19(12):828-37. https://doi.org/10.1093/ molehr/gat065.

37. Rodriguez-Rigau LJ, Smith KD, Tcholakian RK, Steinberger E. Effect of prednisone on plasma testosterone levels and on duration of phases of the menstrual cycle in hyperandrogenic women. Fertil Steril. 1979;32(4):408-13. https://doi.org/10.1016/s0015-0282(16)44296-2.

38. Trott EA, Plouffe L Jr, Hansen K, Hines R, Brann DW, Mahesh VB. Ovulation induction in clomiphene-resistant anovulatory women with normal dehydroepiandrosterone sulfate levels: beneficial effects of the addition of 
dexamethasone during the follicular phase. Fertil Steril. 1996;66(3):484-6. https://doi.org/10.1016/s0015-0282(16)58525-2.

39. Sunkara SK, Coomarasamy A. Androgen pretreatment in poor responders undergoing controlled ovarian stimulation and in vitro fertilization treatment. Fertil Steril. 2011;95(8):e73-4; author reply e75. https://doi.org/10. 1016/.j.fertnstert.2011.04.083.

40. Qin JC, Fan L, Qin AP. The effect of dehydroepiandrosterone (DHEA) supplementation on women with diminished ovarian reserve (DOR) in IVF cycle: Evidence from a meta-analysis. J Gynecol Obstet Hum Reprod. 2017; 46(1):1-7. https://doi.org/10.1016/j.jgyn.2016.01.002.

41. Sunkara SK, Coomarasamy A, Arlt W, Bhattacharya S. Should androgen supplementation be used for poor ovarian response in IVF. Hum Reprod. 2012;27(3):637-40. https://doi.org/10.1093/humrep/der464.

42. Ferraretti AP, La Marca A, Fauser BC, Tarlatzis B, Nargund G, Gianaroli L, ESHRE working group on Poor Ovarian Response Definition. ESHRE consensus on the definition of 'poor response' to ovarian stimulation for in vitro fertilization: the Bologna criteria. Hum Reprod. 2011;26(7):1616-24. https://doi.org/10.1093/humrep/der092.

\section{Publisher's Note}

Springer Nature remains neutral with regard to jurisdictional claims in published maps and institutional affiliations.

- fast, convenient online submission

- thorough peer review by experienced researchers in your field

- rapid publication on acceptance

- support for research data, including large and complex data types

- gold Open Access which fosters wider collaboration and increased citations

- maximum visibility for your research: over $100 \mathrm{M}$ website views per year

At $\mathrm{BMC}$, research is always in progress. 